\title{
Urology \\ PENTAFECTA OUTCOME IN INITIAL 30 CASES OF ROBOT ASSISTED PARTIAL NEPHRECTOMY - OUR EXPERIENCE
}

\section{Dr. Jeevan Kumar}

\section{Dr. Farhana} Zakaria*

\section{Dr. Altaf Khan}

Dr. Lavanya Raghu Sarath $\mathbf{P}$

\section{Dr.} Mujeeburahiman M
Department of Urology, Yenepoya Medical College Hospital, Yenepoya (Deemed to be University), Mangalore, India-575018.

Department of Pathology, Srinivas Institute of Medical Sciences and Research Mukka, Mangalore, India-574146. *Corresponding Author

Department of Urology, Yenepoya Medical College Hospital, Yenepoya (Deemed to be University), Mangalore, India-575018.

Department of Urology, Yenepoya Medical College Hospital, Yenepoya (Deemed to be University), Mangalore, India-575018.

Department of Urology, Yenepoya Medical College Hospital, Yenepoya (Deemed to be University), Mangalore, India-575018.

ABSTRACT Objectives: To study the Pentafecta outcome of initial 30 cases of Robot Assisted Partial Nephrectomy (RAPN) at our institute.

Materials And Methods: A total of 30 patients who underwent Robot assisted partial nephrectomy between January 2016 and June 2018 were prospectively analyzed. The Pentafecta outcome was defined as a warm ischemia time (WIT) of $<25$ min, negative surgical margins, no surgical complications, $>90 \%$ of eGFR preservation \& no CKD upstaging.

Results: All patients successfully underwent robot assisted partial nephrectomy. Median warm ischemia time was 24.5 minutes. One patient with complex tumour had positive surgical margin. There were no Clavien- Dindo Grade IV and V complications. Grade I-III complications were seen in $20 \%$ of patients.

Conclusion: RAPN is safe and effective treatment option for renal tumors and it helps in improving pentafecta outcome.

KEYWORDS : 1.Renal Cell Carcinoma 2.Pentafecta Outcome 3.Partial Nephrectomy 4.Robot Assisted Partial Nephrectom

\section{INTRODUCTION}

Renal Cell Carcinoma (RCC) accounts for $2-3 \%$ of all adult malignant neoplasm with a male predominance $(1.5: 1)$. Peak incidence occurs between 60 and 70 years. Due to increased usage of ultrasound (US) and computed tomography (CT), incidentally diagnosed RCCs have increased. However these tumors are of small size and of lower stage ${ }^{[1]}$. These incidentally detected renal tumors constitute 48 to $66 \%$ of renal tumors. Historically Radical Nephrectomy $[\mathrm{RN}]$ was the treatment for any renal tumors, however Partial Nephrectomy [PN] has now became gold standard for clinically localized small renal masses ${ }^{[2]}$. The main concern with $\mathrm{RN}$ is that it predisposes to $\mathrm{CKD}$, which is potentially associated with morbid cardiovascular events and increased mortality rates. Multiple retrospective series in the past 5 years have compared $\mathrm{PN}$ versus $\mathrm{RN}$ for the management of clinical T1 renal masses, almost uniformly concluding in favor of PN.

Nephron-sparing surgery for the treatment of a renal tumor was first described by Czerny in 1890. A functioning renal remnant of at least $20 \%$ to $30 \%$ of one kidney is necessary to avoid end-stage renal failure, although this presumes good functional status of the remaining parenchyma. Overall preservation of renal function is thus achieved in the great majority of patients with PN, even in patients with traditional imperative indications.

The rates of partial nephrectomy have increased in past decades, comprising about $30 \%$ of all renal surgeries for clinically localized renal masses ${ }^{[3]}$. Robot assisted surgeries have better safety profile and better oncological outcome compared to laparoscopic surgeries.

The feasibility of PN depends on size \& complexity of renal mass and which in turn determines the warm ischemia time. The success of PN depends on oncological and functional outcome. The Trifecta outcome defined as a warm ischemia time (WIT) of $<25 \mathrm{~min}$, negative surgical margins, and no perioperative complications is not a complete outcome assessment tool. With the addition of two more components $90 \%$ estimated glomerular filtration rate preservation and no chronic kidney disease stage upgrading (termed PENTAFECTA) ${ }^{[4]}$ becomes a good assessment tool for success of partial nephrectomy.

Our study aims to assess the PENTAFECTA outcome following
Robotic Assisted Partial Nephrectomy [RAPN] in patients with clinically localized RCC.

\section{MATERIALSAND METHODS:}

30 patients who underwent robot assisted partial nephrectomy (RAPN) between January 2016 and June 2018 were included in our prospective study. All participants gave informed written consent. All surgeries were performed by the same urologist with the assistance of two other urologists of the team. All cases were clinically localised RCC's.

Collected data included Patient demographics [age, gender, body mass index (BMI)]; Preoperative evaluation of [baseline renal function, chronic kidney disease (CKD) stage, TNM staging, R.E.N.A.L. nephrometry score] ${ }^{[5]}$, Intraoperative data [operative time, warm ischemia time (WIT), estimated blood loss (EBL)], Perioperative complications (Clavien- Dindo Classification of Surgical Complications) ${ }^{[6]}$.

As well as pathology findings (American Joint Cancer Committee staging and margin status) ${ }^{[7]}$, and long-term functional outcomes were assessed using eGFR (Estimated GFR) ${ }^{[8]}$.

(eGFR) calculated using the Cockroft-Gault formula. Statistical analysis was performed using the chi-square test and SPSS v21 software.

\section{Inclusion Criteria -}

All patients with clinically localized RCC admitted from January 2016 to June 2018 regardless of age and sex were included in the study.

\section{Exclusion Criteria-}

Locally Advanced and metastatic RCC, concomitant cancers, severe systemic illness.

\section{RESULTS:}

About 30 patients underwent partial nephrectomy with a median follow-up of 18 months, with 7 patients having a follow-up of $>24$ months. 
Volume - 10 | Issue - 12 | December - 2020 | PRINT ISSN No. 2249 - 555X | DOI : 10.36106/ijar

Table 1 The Cohort's Characteristics Are Summarized.

\begin{tabular}{|l|l|}
\hline COHORT CHARACTERISTICS & \\
\hline AGE [ MEAN ,RANGE] & $56(38-72)$ \\
\hline SEX (M/F) & $22 / 8$ \\
\hline TUMOR SIZE & $3.8(1.7 \mathrm{~cm}-6.6 \mathrm{~cm})$ \\
\hline T1a & 19 \\
\hline T1b & 11 \\
\hline R.E.N.A.L. Nephrometry score, (\%) & \\
\hline LOW (4-6) & $65 \%$ \\
INTERMEDIATE (7-9) & $25 \%$ \\
HIGH (10-12) & $10 \%$ \\
\hline Preoperative eGFR, $\mathrm{mL} / \mathrm{min} / 1.72 \mathrm{~m}^{2}$, (mean) & 78 \\
\hline
\end{tabular}

Table 2: Represents The Operative And Perioperative Characteristics

\begin{tabular}{|l|l|}
\hline WARM ISCHEMIA TIME (mean) & $24.5(16-30)$ \\
\hline MEAN OPERATIVE TIME, min & $184(142-230)$ \\
\hline ESTIMATED BLOOD LOSS, ML & $160(100-350)$ \\
\hline Clavien-Dindo grade of complications, $\mathrm{n}(\%)$ & \\
\hline I & $3(10 \%)$ \\
\hline II & $3(10 \%)$ \\
\hline III & $1(3.3 \%)$ \\
\hline IV & 0 \\
\hline V & 0 \\
\hline
\end{tabular}

Table 3: Represents The Pentafecta Outcome In Overall

\begin{tabular}{|l|l|l|}
\hline COMPONENT & $\begin{array}{l}\text { OUTCOME } \\
\text { ACHIEVED IN }\end{array}$ & PERCENTAGE \\
\hline (WIT) of $<25$ min & $22(30)$ & $73.33 \%$ \\
\hline Negative surgical margins & $29(30)$ & $96.6 \%$ \\
\hline $\begin{array}{l}\text { NO Complications [Clavien- } \\
\text { Dindo } \geq 2 \text { ] }\end{array}$ & $26(30)$ & $86.6 \%$ \\
\hline eGFR $>90 \%$ at end of 1 yr & $25(30)$ & $83.3 \%$ \\
\hline No up gradation of CKD status & $26(30)$ & $86.6 \%$ \\
\hline $\begin{array}{l}\text { COMPLETE PENTAFECTA } \\
\text { ACHIEVEMENT }\end{array}$ & PERCENTAGE \\
\hline 22(30) & $73.3 \%$ \\
\hline
\end{tabular}

Table 4: Represents The Pentafecta Outcome Separately In T1a \& T1b Lesions

\begin{tabular}{|c|c|c|c|c|}
\hline COMPONENT & \multicolumn{2}{|c|}{$\begin{array}{l}\text { OUTCOME } \\
\text { ACHIEVED IN } \\
\text { T1a(19) }\end{array}$} & \multicolumn{2}{|c|}{$\begin{array}{l}\text { OUTCOME } \\
\text { ACHIEVED IN } \\
\text { T1b(11) } \\
\end{array}$} \\
\hline$($ WIT) of $<25 \mathrm{~min}$ & \multicolumn{2}{|c|}{$16(19)-[84.21 \%]$} & \multicolumn{2}{|c|}{$7(11)-[63.63 \%]$} \\
\hline $\begin{array}{l}\text { Negative surgical } \\
\text { margins }\end{array}$ & \multicolumn{2}{|c|}{$19(19)-[100 \%]$} & \multicolumn{2}{|c|}{$10(11)-[90.9 \%]$} \\
\hline $\begin{array}{l}\text { NO } \\
\text { Complications } \\
\text { [Clavien- Dindo } \\
\geq 2 \text { ] }\end{array}$ & \multicolumn{2}{|c|}{$17(19)-[89.47 \%]]$} & \multicolumn{2}{|c|}{$9(11)-[81.8 \%]$} \\
\hline $\begin{array}{l}\text { eGFR }>90 \% \text { at } \\
\text { end of } 1 \mathrm{yr}\end{array}$ & \multicolumn{2}{|c|}{$17(19)-[89.47 \%]]$} & \multicolumn{2}{|c|}{$8(11)-[72.72 \%]$} \\
\hline $\begin{array}{l}\text { No up gradation } \\
\text { of CKD status }\end{array}$ & \multicolumn{2}{|c|}{$18(19)-[94.7 \%]$} & \multicolumn{2}{|c|}{$8(11)-[72.72 \%]$} \\
\hline \multicolumn{2}{|c|}{$\begin{array}{l}\text { COMPLETE PENTAFECTA } \\
\text { ACHIEVEMENT }\end{array}$} & \multicolumn{2}{|c|}{ T1a } & T1b \\
\hline \multicolumn{2}{|c|}{$22(30)-[73.33 \%]$} & \multicolumn{2}{|c|}{ 15(19)-[78.94\%] } & $9(11)-[63.63 \%]$ \\
\hline
\end{tabular}

\section{DISCUSSION:}

In this modern era renal masses are increasingly treated with NSS, whenever feasible. The R.E.N.A.L. (radius, exophytic/endophytic properties, and nearness of tumor to the collecting system or sinus in millimeters, anterior/posterior location relative to polar lines) nephrometry scoring system was recently introduced as an objective reproducible means to describe salient renal tumor anatomy ${ }^{7}$. Since its introduction, the RENAL nephrometry scoring system has been shown to provide important preoperative and perioperative information used to predict long term outcomes and is increasingly being incorporated into the management of renal tumour.

Minimally invasive approaches, e.g. robotic assisted partial nephrectomy are being increasingly used ${ }^{[5,6]}$. RAPN has been shown to be safe and effective, even for complex renal tumors ${ }^{[8]}$. The advantages of the robotic approach include better ergonomics, enhanced vision, and short learning curve.

The concept of Trifecta outcomes in partial nephrectomy was first introduced by Gill et al ${ }^{[9]}$. In trifecta outcome 3 key outcomes of negative cancer margin, warm ischemia time (WIT) $<25 \mathrm{~min}$ and no urological complications are simultaneously realized. With the addition of two more components $90 \%$ estimated glomerular filtration rate preservation and no chronic kidney disease stage upgrading (termed PENTAFECTA) becomes a good assessment tool for success of partial nephrectomy.

The rate of Pentafecta achievement in RAPN was $73.33 \%$ ( overall), $78.94 \%$ (T1a) \& 63.63\% (T1b). Our contemporary Laparoscopic partial Nephrectomy (LPN) case series (18n) during the same period were also evaluated for Pentafecta outcome which was $66.2 \%$ (overall), 70.4\% (T1a) \& 60.2\% (T1b). The major limitation in (LPN) series was because of increased warm ischemic time (mean $28 \mathrm{~min}$ ) where it was 24.5 in RAPN. The major limitation for pentafecta achievement in our study was found to warm ischemia time which in turn affected complexity of renal mass. Cases with increased warm ischemia time were found to have high nephrometry score therefore RENAL score is predictor of pentafecta outcome achievement.

Table 5: Compares The Pentafecta Outcome In Other Studies (4) (9) (10)

\begin{tabular}{|l|l|l|}
\hline Case series & T1a & T1b \\
\hline Our study (30) & $78.94 \%$ & $63.63 \%$ \\
\hline Kang ${ }^{\text {cetal(362) }}$ & $45.7 \%$ & $25.9 \%$ \\
\hline Dae Keun kim(277) & $38.3 \%$ & $26.7 \%$ \\
\hline Zargar,etal (1831) & $38.4 \%$ & $28.9 \%$ \\
\hline
\end{tabular}

The rates of Pentafecta achievement in various studies are shown in the table5. Compared with other studies the rate of pentafecta achievement was found superior in our study.

\section{Limitations:}

The limitations of our study were small Sample size, most of the patients in our case series are having Low Nephrometry score (65\%). The medical premorbid conditions (diabetes, hypertension) which affect the functional outcome ( eGFR) were not matched while comparing other studies.

\section{CONCLUSION:}

RAPN is a safe and effective treatment option for renal tumors whenever feasible.

\section{REFERENCES:}

1. Kato, M., et al. Natural history of small renal cell carcinoma: evaluation of growth rate, histological grade, cell proliferation and apoptosis. JUrol, 2004. 172: 863 .

2. Kunath F, Schmidt S, Krabbe LM, Miernik A, Dahm P, Cleves A, WaltherM, Kroeger N Partial nephrectomy versus radical nephrectomy for clinical localised renal masses. Cochrane Database of Systematic Reviews 2017;5: CD012045.

3. Bianchi M, Becker A, Abdollah F, Trinh QD, Hansen J, Tian Z, et al. Rates of open versus laparoscopic and partial versus radical nephrectomy for Tla renal cell carcinoma: A population-based evaluation. International Journal of Urology 2013;20(11):1064-107.

4. Zargar H, Allaf ME, Bhayani S, Stifelman M, Rogers C, Ball MW, Larson J, Marshall S Kumar R, Kaouk JH. Trifecta and optimal perioperative outcomes of robotic and laparoscopic partial nephrectomy in surgical treatment of small renal masses: a multiinstitutional study. BJU Int. 2015 Sep;116(3):407-14

5. Rosaleen B. Parsons, Daniel Canter, Alexander Kutikov, Robert G. Uzzo. RENAL Nephrometry Scoring System: The Radiologist's Perspective. (2012) American Journal of Roentgenology. 199(3): W355-W359.

6. Clavien PA, Barkun J, de Oliveira ML, Vauthey JN, Dindo D, Schulick RD, de Camtibañes E, Pekolj J, Slankamenac K, Bassi C, Graf R, Vonlanthen R, Padbury R, five-year experience. Ann Surg. 2009 Aug; 250(2):187-96.

7. Swami, Umang et al. "Revisiting AJCC TNM staging for renal cell carcinoma: quest for improvement." Annals of translational medicine vol. 7,Suppl 1 (2019): S18. doi:10.21037/atm.2019.01.50

8. Cockcroft DW, Gault H. Prediction of creatinine clearance from serum creatinine. Nephron 1976; 16:31-41.

9. Kim DK, Kim LHC, Raheem AA, Shin TY, Alabdulaali I, Yoon YE, et al. (2016) Comparison of Trifecta and Pentafecta Outcomes between T1a and T1b Renal Masses following Robot-Assisted Partial Nephrectomy (RAPN) with Minimum One Year Follow Up: Can RAPN for T1b Renal Masses Be Feasible? PLoS ONE 11(3): e0151738. https://doi.org/10.1371/journal.pone. 0151738

10. Kang M, Gong IH, Park HJ, Sung HH, Jeon HG, Jeong BC, Jeon SS, Lee HM, Choi HY, Il Seo S. Predictive Factors for Achieving Superior Pentafecta Outcomes Following Robot-Assisted Partial Nephrectomy in Patients with Localized Renal Cell Carcinoma. J Endourol. 2017 Dec; 31(12):1231-1236. 